\title{
Construction of a temperature controlled Rheo-NMR measuring cell - Influence of fluid dynamics on PMMA-polymerization kinetics
}

\author{
E. Laryea ${ }^{\text {a }}$, N. Schuhardt ${ }^{\text {a }}$, G. Guthausen ${ }^{\text {b, }}{ }^{*}$, T. Oerther ${ }^{\text {c }}$, M. Kind ${ }^{\text {a }}$ \\ a Karlsruhe Institute of Technology, Institute of Thermal Process Engineering, 76131 Karlsruhe, Germany \\ ${ }^{\mathrm{b}}$ Karlsruhe Institute of Technology, Institute for Mechanical Process Engineering and Mechanics and Institute for Water Chemistry and Water Technology, \\ 76131 Karlsruhe, Germany \\ c Bruker BioSpin GmbH, 76287 Rheinstetten, Germany
}

Keywords:

Rheo-NMR

Fluid dynamics

Polymerization kinetics

Free radical polymerization

\begin{abstract}
A B S T R A C T
The aim of this work is it to develop a measuring method which allows to detect and quantify the in fluence of the fluid dynamics on polymerization kinetics. Such a dependency has been claimed previ ously. However, yet there is no analytical method available which gives insight into the phenomena observed on a molecular level. Rheo NMR is a technique, which is particularly suitable to analyze such phenomena. Hence, a temperature controlled Rheo NMR measuring cell was constructed. The validation of the concept is demonstrated on a model system, the free radical polymerization of methyl methacrylate.
\end{abstract}

\section{Introduction}

Polymerization poses special challenges to reaction engineering, not at least because of the dependence between the polymer properties and the process conditions. Both industry and research aim for a comprehensive control and optimization of polymeriza tion processes. Important quality parameters are, for example consistent product properties and tailor made polymers. This im plies the importance of simulation and prediction of polymer processes, especially for the prediction of end use properties. Knowledge is required about the process parameters influencing the polymerization and its kinetics. One of these influencing pa rameters is fluid dynamics. 1980 Kumar et al. [1] reported on an apparatus, which combines a dilatometer and a rheometer. An in crease of the reaction rate of the free radical polymerization (FRP) of methyl methacrylate (MMA) was observed inside such an apparatus and was attributed to an increase of the radical efficiency caused by shearing the reaction mixture during the polymerization. Other studies show that the use of a spinning disc reactor also af fects polymerizations due to the shear of the reaction mixture

\footnotetext{
* Corresponding author.

E-mail address: gisela.guthausen@kit.edu (G. Guthausen).
}

[2-5]. The term "hydrodynamic activation" was established 2011 by Liu et al. [6] and describes the promoting influence of process parameters on polymer product properties. A batch polymerization was carried out in a Taylor Couette reactor (TCR). It was found that if the FRP of MMA takes place in the gap between two concentric cylinders and the inner one rotates, the molecular weight distri bution as well as the monomer conversion will depend on the angular velocity of the inner cylinder. The influence of hydrody namics on kinetics was attributed to a change of the propagation respectively termination of the polymers. In summary, different approaches have been explored to investigate the influence of fluid dynamics on polymerization kinetics but there is still no detailed explanation. Without a detailed knowledge about the influence of fluid dynamics on polymerizations it will neither be possible to model nor to make good use of it. To elucidate the phenomena observed a measuring method is required, which allows reaction monitoring under conditions of well defined fluid flow and defor mation. A realisation could be Rheo NMR [7]. On the one hand, nuclear magnetic resonance (NMR) spectroscopy offers the possi bility to gain information about a fluid on a molecular level, for example about its chemical composition. On the other hand the Rheo NMR measurement enables the determination of fluid flow by magnetic resonance imaging (MRI). For example Seymour et al. [8] showed that the Taylor vortex flow in a Couette cell can be 
measured by PGSE NMR data. For these reasons a temperature controlled Rheo NMR measuring cell was constructed which al lows in situ monitoring of polymerization reactions at typical re action parameters. Apart from the chemical composition and the flow field, the method proposed reveals hints on whether the fluid dynamics changes the polymer conformation and if this affects the reaction kinetics. To demonstrate this methodology FRP of MMA was used as an example system.

\section{Theory}

\subsection{Free radical polymerization}

The model system chosen is the free radical solution polymer ization of MMA. Basically, FRP can be divided into three main mechanisms: initiation, propagation and termination. The first step describes the decomposition of an initiator molecule into two radicals by the supply of energy. Those radicals react with mono mers and build radicalized monomers. In the second step, polymers form and propagate in molecular weight due to addition of further monomers. The end of propagation is the termination step. One possibility for a growing polymer chain to terminate is the reaction with another radicalized polymer chain by combination. As a result, one long non radicalized polymer chain is formed. In this work chain transfer reactions, which means the transfer of a radical of a growing chain to another molecule (solvent, monomer), is not considered. The monomer conversion $X_{M}(t)$ describes the molar fraction of reactants (index $M$ for monomer) at reaction time $t$, which has reacted to the product. In a batch polymerization, the theoretical course of monomer conversion is

$X_{M}(t) \equiv \frac{n_{M, 0} \quad n_{M(t)}}{n_{M, 0}} \quad 1 \quad \exp \left(\frac{2 k_{1}}{k_{d}} \cdot \sqrt{ } c_{I, 0}\left(1 \quad \exp \left(\frac{k_{d} \cdot t}{2}\right)\right)\right)$

where $n_{M, 0}[\mathrm{~mol}]$ is the initial amount of monomer and $n_{M(t)}[\mathrm{mol}]$ is the amount of monomer at the time $t[s] . k_{d}\left[s^{-1}\right]$ is the decompo sition rate constant and $c_{I, 0}\left[\mathrm{~mol} \cdot \mathrm{l}^{-1}\right]$ is the initial initiator con centration [9]. The overall reaction rate constant $k_{1}\left[l^{0.5} \cdot \mathrm{s}^{-1} \cdot \mathrm{mol}^{-0.5}\right]$ summarises all individual rate constants, i.e. $k_{d}\left[\mathrm{~s}^{-1}\right]$ (decomposition), $k_{p}\left[l \cdot \mathrm{s}^{-1} \cdot \mathrm{mol}^{-1}\right]$ (propagation) and $k_{t}\left[l \cdot s^{-1} \cdot \mathrm{mol}^{-1}\right]$ (termination), and the initiator efficiency $f$ as well: $k_{1} \frac{k_{p}}{\sqrt{ } k_{t}} \cdot \sqrt{2 \cdot f \cdot k_{d}}$

$f$ describes the fraction of initiator molecules, which effectively start the growth of a polymer chain. A detailed description of the free radical polymerization kinetics can be obtained from Ref. [9].

\subsection{Fluid dynamics between two concentric cylinders}

A basic prerequisite for a quantitative insight into the influence of fluid dynamics on polymerization kinetics is that the experi ments have to take place under variable, but well defined flow conditions. Two concentric cylinders, i.e. a Couette cell, are used because the flow profile in the gap between these rotating cylinders is well characterised $[10,11]$. Furthermore, this system has a ho mogeneous energy dissipation along the symmetry axis, i.e. the rotation axis of the cylinders. The flow stability can be described by the dimensionless rotational Reynolds number $R e_{\Phi}$.

$\operatorname{Re}_{\Phi} \quad \frac{\omega \cdot R_{i} \cdot d}{\nu}$

Here, $\omega\left[s^{-1}\right]$ is the angular velocity and $R_{i}[m]$ the radius of the inner cylinder, $d[\mathrm{~m}]$ the gap width and $\nu\left[\mathrm{m}^{2} \cdot \mathrm{s}^{-1}\right]$ the kinematic viscosity of the fluid. In literature, the Taylor number $\mathrm{Ta}$ (Eq. (4)) is often used instead of the rotational Reynold number to characterise the flow state.

Ta $\operatorname{Re}_{\Phi} \sqrt{\frac{d}{R_{i}}}$

Four main flow regimes are known between two cylinders with a rotating inner and resting outer cylinder (Fig. 1). The laminar flow is a shear flow with linear profile. When a critical Reynolds number is exceeded, the flow changes into the toroidal Taylor vortex flow. With further increase of the Reynolds number the flow regime changes into the wavy vortex flow, in which a wavy movement of each toroidal vortex superimposes the vortex flow. The fourth main flow regime is the turbulent flow. In this flow state no waves are detectable. Andereck et al. [12] give a detail explanation of the different flow regimes. The critical rotational Reynolds numbers $R e_{\Phi, \text { crit }}$ describe the turnover points of the different flow regimes

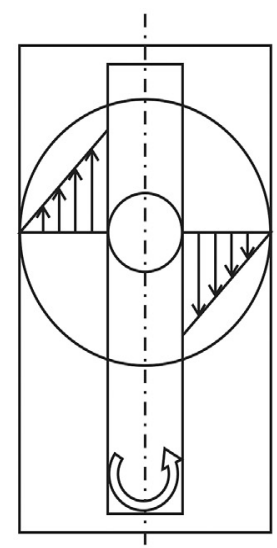

a)

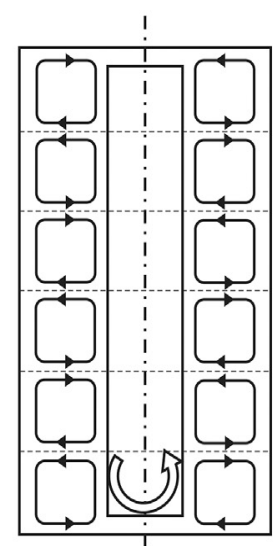

b)

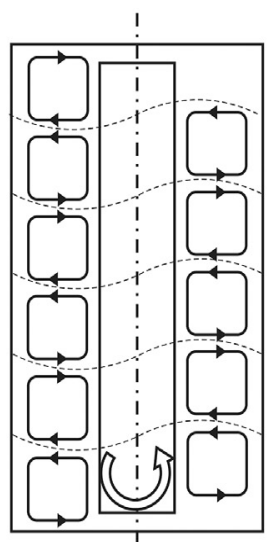

c)

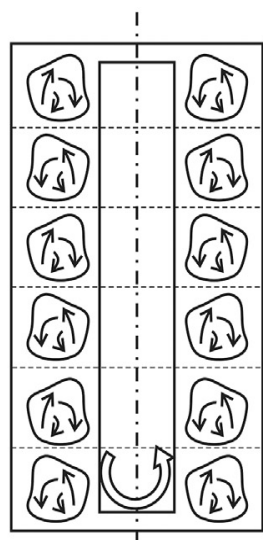

d)

Fig. 1. Main flow regimes between to concentric cylinder if the inner cylinder rotates and the outer cylinder rests in order of appearance with increasing rotational Reynolds number a) Laminar (rheometric) flow b) Taylor vortex flow c) Wavy vortex flow d) turbulent flow, according to Racina [19]. 
and depend in general on the radius ratio $\eta$ (Eq. (5)) and the aspect ratio $\Gamma$ (Eq. (6)).

$$
\begin{aligned}
& \eta \frac{R_{i}}{R_{i}+d} \\
& \Gamma \frac{L}{d}
\end{aligned}
$$

With $L[m]$ being the length of the gap. In some publications, the apparent shear rate is used to quantify the process conditions and the resulting stress on the fluid.

$\dot{\gamma} \frac{\omega \cdot R_{i}}{d}$

Formally, the use of shear rate is not correct in the unstable flow regimes of Taylor vortex, Wavy vortex and turbulent flow. Never theless, also in these cases and not only in the laminar flow regime, the apparent shear rate is assumed to be a useful fluid dynamic parameter since it is not a function of the fluid properties.

\section{Construction of a temperature controlled Rheo-NMR measuring cell}

In this work we used a $400 \mathrm{MHz}$ spectrometer with a $25 \mathrm{~mm}$ micro imaging probe and the Bruker Rheo NMR system [13]. The measuring cell (Fig. 2) consists of two concentric cylinders made out of polyether ether ketone (PEEK). The outer diameter of the inner cylinder is $17 \mathrm{~mm}$, and the inner diameter of the outer cyl inder is $19 \mathrm{~mm}$. Thus, the resulting gap width is $1 \mathrm{~mm}$ and ac cording to equation (5) the radius ratio is $\eta \quad 0.895$. The length of the gap is $L \quad 59 \mathrm{~mm}$, hence the aspect ratio of the construction is $\Gamma \quad 59$. The critical rotational Reynolds numbers, which describe the change of the flow regimes, are independent of the cylinder length and depend only on the radius ratio $\eta$ for an aspect ratio $\Gamma>40$ [9]. This relation simplifies the interpretation of the flow regimes set in the experiments. Furthermore, no distinction was made between the Taylor vortex flow and the wavy vortex flow, because Andereck et al. [11] showed that the Reynolds number region, in which this flow regime exists, is very small. The critical Reynolds number which describes the turnover between the laminar flow and the Taylor vortex respectively the wavy vortex flow is $R e_{\Phi, \text { crit }} 129$ and was determined with the equation given by Esser and Grossmann [14].

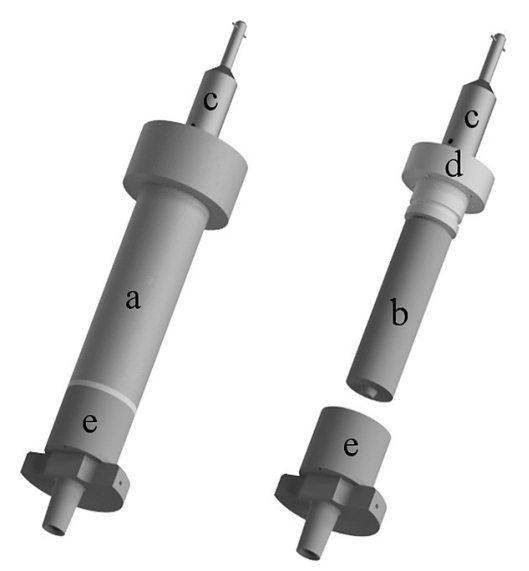

Fig. 2. Design drawing of the Rheo-NMR measuring cell; left: with outer cylinder, right without outer cylinder, a) outer cylinder, b) inner cylinder, c) coupling, d) bearing, e) heating adapter.

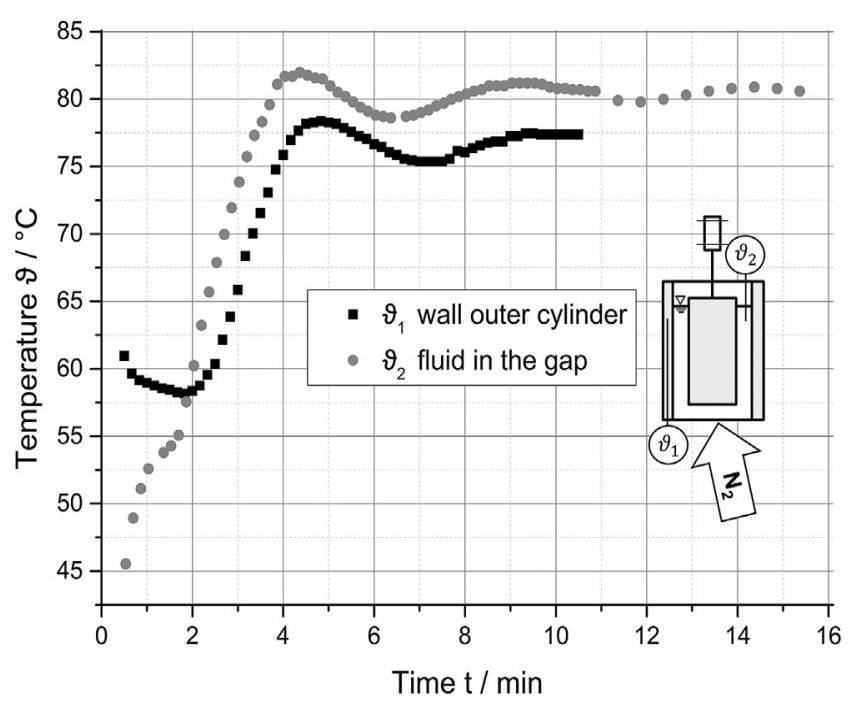

Fig. 3. Temperatures measured by the thermocouple in the wall $\left(\vartheta_{1}\right)$ and the fluid (water) in the gap $\left(\vartheta_{2}\right)$ without rotting the inner cylinder. The measuring cell was preheated and the volume flow of the hot nitrogen was $1200 \mathrm{l} / \mathrm{h}$.

The concentric arrangement of the two cylinders is ensured by using bearings. The temperature of the reaction system has to be well controlled and kept at a constant value. To this end, hot ni trogen passes a gas distributer, which spreads and distributes the gas stream into small axial channels in the wall of the outer cyl inder. The small gap width of $1 \mathrm{~mm}$ inhibits a direct, in situ mea surement of the temperature inside the measuring cell, because a thermocouple in the gap would disturb the fluid flow. Instead, the thermocouple was mounted into one of the channels in the wall of the outer cylinder. The thermocouple was insulated to the heating gas stream (to avoid heat conduction). In order to obtain a corre lation between the temperature $\vartheta_{1}$ measured by the thermocouple in the wall and the fluid temperature in the gap, a calibration was carried out. In a static experiment without rotation a second ther mocouple (temperature $\vartheta_{2}$ ) was put into the fluid in the gap to determine the temperature deviation, which is due to the different measurement positions. Corresponding to the results (Fig. 3), the setpoint temperature of the thermocouple in the wall was set to $\vartheta_{1} \quad 76.8{ }^{\circ} \mathrm{C}$ for the following experiments to achieve a reaction temperature of $80^{\circ} \mathrm{C}$.

\section{Model experiment}

The free radical polymerization of methyl methacrylate (MMA) in a solvent was chosen as model reaction, to test the functionality of the construction and the experimental concept. The polymeri zation was carried out in the Rheo NMR measuring cell. 2,2 azoisobutyronitrile (AIBN) and xylene were used as initiator and solvent, respectively. The experiments were carried out with initial reaction mixtures consisting of $50 \mathrm{wt}$. \% MMA, $0.5 \mathrm{wt}$. \% AIBN and p xylene. The structural formula of MMA and polymethyl meth acrylate (PMMA) show the reaction mechanism (Fig. 4). After stir ring the reaction mixture at ambient temperature for about $1 \mathrm{~min}$<smiles>COC(=O)/C(C)=C\CCC(C)(C)CC(C)(C)C</smiles>

Fig. 4. Structural formula of MMA and PMMA. 
to homogenize it, it was filled into the preheated Rheo NMR cell. Afterwards, the Rheo NMR cell was installed in the micro imaging probe, inserted into the spectrometer and started to rotate the in ternal cylinder at the preset rotational frequency of $144 \mathrm{rpm}$. This procedure took about $1 \mathrm{~min}$. The recording of the reaction time was started when the mixture was filled into the cell. The rotational frequency corresponds to an apparent medium shear rate of $\dot{\gamma} 128 \mathrm{~s}^{-1}$. The data recording was stopped after a total reaction time $t$ of about 70 min.

\section{Materials and NMR experimental details}

MMA (99.0\%) was purchased from VWR International GmbH, p xylene ( $\geq 99.0 \%$ ) from Carl Roth $\mathrm{GmbH}$, and 2,2 azoisobutyronitrile (AIBN; $\geq 98.0 \%$ ) from Sigma Aldrich. The polymerization progress was measured by ${ }^{1} \mathrm{H}$ spectra, acquired in a $1 \mathrm{D}$ single pulse NMR experiment (multi $\mathrm{zg}$ pulse sequence; relaxation delay $D_{1} 500 \mathrm{~ms}$, number of scans $N S 4$, pre acquisition delay DE $6.5 \mu \mathrm{s}$ ), on an AVANCE HDIII $400 \mathrm{WB}$ spectrometer at Bruker, Germany, equipped with the Rheo NMR system. The measuring cell was installed into a MicWB40 probe. This probe allowed for gra dients up to about $1.5 \mathrm{~T} / \mathrm{m}$. Data processing was performed within self written MATLAB ${ }^{\circledR}$ scripts.

\section{Results and discussion}

The processing of the measurement data is challenging when aiming for a quantitative description of in situ reaction kinetics. In this work, the monomer conversion was extracted from the ${ }^{1} \mathrm{H}$ NMR spectra which were acquired during the reaction taking place under shear. The spectra were modeled and approximated by non linear fits, and subsequently, the overall reaction rate constant $k_{1}$ was calculated after fitting the model parameter of the kinetic equation, see Eq. (1), to the data.

There are a few special aspects due to the special Rheo NMR cell and the measuring procedure, which have to be taken into account. The essential part of the ${ }^{1} \mathrm{H}$ NMR spectrum of the reaction mixture (Fig. 5) shows two peaks at a chemical shift of $\delta \quad 6.1 \mathrm{ppm}$ (a) and $\delta \quad 5.5 \mathrm{ppm}$ (b). They represent the ${ }^{1} \mathrm{H}$ atoms of the monomer MMA, which are part of the methylene group $\left(\mathrm{CH}_{2}\right)$. The methyl group of the solvent $\mathrm{p}$ xylene causes the peak at $\delta \quad 7.0$ (c). The

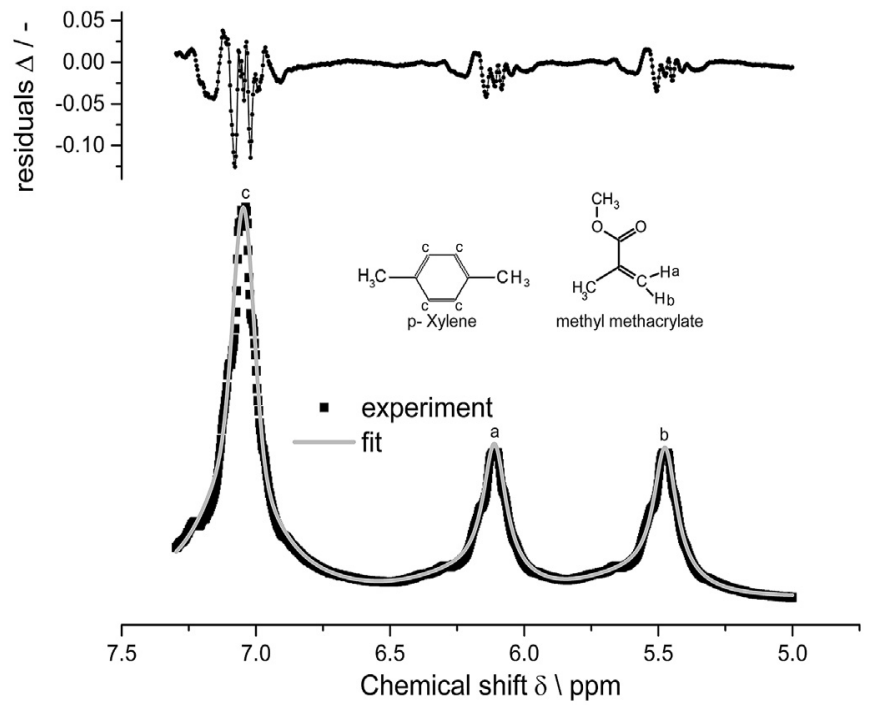

Fig. 5. Essential part of the ${ }^{1} \mathrm{H}-\mathrm{NMR}$ spectra of reaction mixture with the initial composition of $50 \mathrm{wt} \% \mathrm{MMA}, 49.5 \mathrm{wt} \% \mathrm{p}$-xylene and $0.5 \mathrm{wt} \%$ AIBN at $80^{\circ} \mathrm{C}$ under shear $(\gamma \quad 1281 / \mathrm{s}$ ) in the Rheo-NMR measuring cell after a reaction time of about $20 \mathrm{~min}$. peaks are very broad, induced by the large diameter and the shape of measuring cell, which deviates obviously from the ideal sample geometry in a standard $5 \mathrm{~mm}$ sample tube. The reaction mixture, which generates the signal, is distributed over the small gap with a big diameter. Thus, the inhomogeneity of the static magnetic field is pronounced and is responsible for the broadening of the peaks. Nevertheless, the monomer conversion was quantified by the nu merical analysis of peaks a) and b), by line fitting. Both peaks and, consequently, their peak areas decrease with progressing poly merization because the double bond $\mathrm{C}=\mathrm{C}$ opens and the ${ }^{1} \mathrm{H}$ atoms of the methyl group shift to a different chemical shift. Due to the large line width, the data were modeled by Pseudo Voigt functions, the parameters of which were determined using a nonlinear least squares solver. The overall fit and the normalized divergence be tween the experimental data and the fit give an impression about the achieved quality (Fig. 5). Furthermore, the fit is in good agreement with the experimental data, because the normalized residuals are very small. As the Rheo NMR measurements usually are executed in an unlocked way, spectral alignment was per formed separately to correct for the observed field drift during measurement time, i.e. the reaction time. Without the alignment, the fits of the peaks would change drastically with time since the complexity of the fit requires tight boundary conditions. Wrong values for the MMA concentrations and therefore wrong $k_{1}$ values would result. The third challenge being evident when comparing spectra at different reaction times is that the peak shape changes with progressing polymerization. The change is particularly evident when considering the $\mathrm{p}$ xylene (peak $\mathrm{c}$ in Fig. 5). The solvent does not take part in the reaction. Accordingly, its peak area should stay constant during the polymerization because it is proportional to the concentration. The spectra indicate a decrease of the area of peak c with the reaction time. The reason of this observation is that the chemical environment alters with the production of polymers. To eliminate this effect the area ratio $\alpha$ of the sum of peak a and $b$ (monomer MMA) $A_{M M A}$ is referenced to the area of peak c (solvent, $\mathrm{p}$ xylene) $A_{\text {Xylene }}$ to quantify the monomer conversion (Eq. (8)).

$\alpha(t) \frac{A_{M M A}(t)}{A_{\text {Xylene }}(t)}$

The shapes of all peaks are assumed to change the same way. The data, i.e. area ratio are plotted as a function of reaction time (Fig. 6). As expected, the area ratio shows a decrease with time

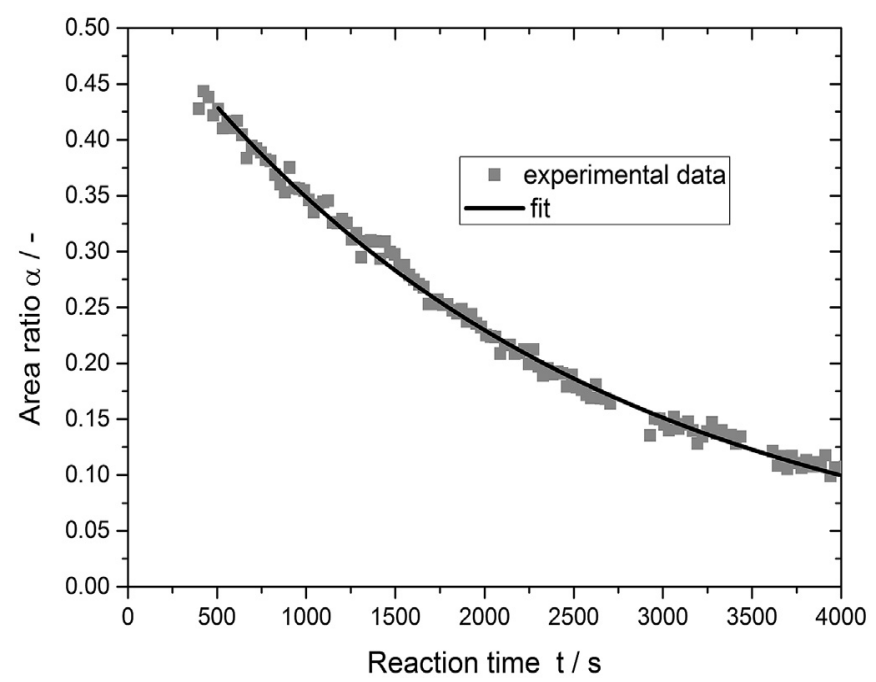

Fig. 6. The calculated area ratio (Eq. (9)) vs. reaction time; process parameters: $\vartheta \quad 80^{\circ} \mathrm{C}$, $\gamma \quad 1281 / \mathrm{s}$, initial composition 50 wt\% MMA, 49.5 wt\% p-xylene 0.5 wt\% AIBN. 


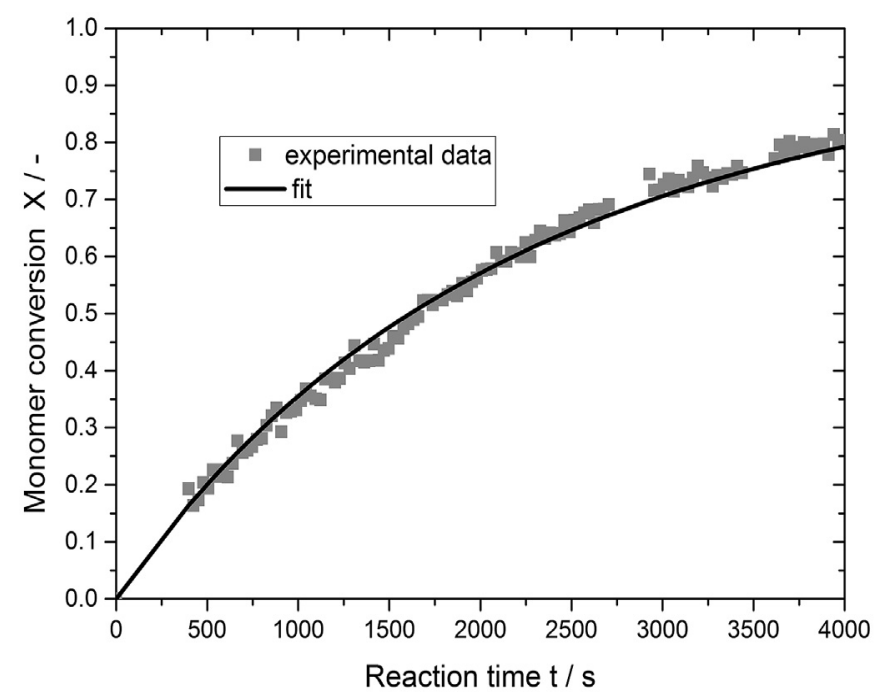

Fig. 7. Monomer conversion vs. reaction time, process parameters: $\mathrm{T} 80^{\circ} \mathrm{C}$, $\gamma \quad 1281 / \mathrm{s}$, initial composition $50 \mathrm{wt} \% \mathrm{MMA}, 49.5 \mathrm{wt} \%$ p-xylene $0.5 \mathrm{wt} \%$ AIBN.

Table 1

Kinetic constants of the free radical polymerization of MMA respectively parameters of the initiator AIBN at $T \quad 80^{\circ} \mathrm{C}$.

\begin{tabular}{lll}
\hline Kinetic constant & Value & Reference \\
\hline$f[-]$ & 0.6 & {$[9]$} \\
$k_{d}\left[\frac{1}{s}\right]$ & $1.5310^{3}$ & {$[16]$} \\
$k_{p}\left[\frac{l}{\mathrm{~mol} \cdot \mathrm{s}}\right]$ & 1317 & {$[17]$} \\
$k_{t}\left[\frac{l}{\mathrm{~mol} \cdot \mathrm{s}}\right]$ & $4.3610^{7}$ & {$[18]$} \\
\hline
\end{tabular}

since the monomer concentration declines with the progress of polymerization. Unfortunately, it is currently not possible to ac quire reliable data at the very beginning of the polymerization. The filling of the pre heated measuring cell outside the spectrometer causes an immediate start of the reaction, without the chance to measure. For the calculation of the monomer conversion the value of the area ratio at the beginning $\left(\begin{array}{ll}t & 0\end{array}\right)$ is necessary (Eq. (10)), however. Hence, the area ratio was back extrapolated with an exponential function to obtain the initial area ratio.

$$
\alpha\left(\begin{array}{ll}
t & 0) \\
0.53
\end{array}\right.
$$

In Eq. (10) the monomer conversion is assumed to be identical to area ratio definition (Cf. Eq. (1)).

$$
X_{M}(t) \quad \frac{\alpha(t \quad 0) \quad \alpha(t)}{\alpha(t \quad 0)}
$$

The monomer conversion derived from the Rheo NMR experi ments shows the expected behavior (Fig. 7). To determine the overall reaction rate constant $k_{1}$ the parameters of Equation (1) were fitted to the experimental data (Fig. 7) by using the least

Table 2

Over all reaction rate constant for the free radical polymerization of MMA with the initiator AIBN at $T \quad 80^{\circ} \mathrm{C}$.

\begin{tabular}{ll|l|}
\hline & Reaction rate constant $k_{1}$ & $\frac{l^{05}}{s \cdot \text { mol05 }}$ \\
\hline Rheo NMR measurement & $2.7110^{3}$ \\
With shear rate dependency & $2.0210^{3}$ \\
Without shear rate dependency & $2.6610^{3}$ \\
\hline
\end{tabular}

squares method and $k_{d}$ from literature (Table 1 ). The initial initiator concentration amounted to $C_{I, 0} \quad 2.6 \cdot 10^{-2} \mathrm{~mol} / \mathrm{l}$ and the reaction rate constant was determined to $k_{1} \quad 2.71 \cdot 10^{-3} l^{0.5} \mathrm{~mol}^{-0.5} \cdot \mathrm{s}^{-1}$.

To validate this data of the reaction rate constant it was compared with data of Liu [15]. Liu investigated the same model system and described the termination rate constant $k_{t}$ as a function of the shear rate (Eq. (11)).

$$
k_{t} \quad 5.1 \cdot 10^{4} \cdot \dot{\gamma}+8.71 \cdot 10^{7}
$$

A shear rate of $\dot{\gamma} \quad 128 s^{-1}$ results in a termination rate constant of $k_{t} \quad 7.51 \cdot 10^{7} \mathrm{l} \cdot \mathrm{s}^{-1} \cdot \mathrm{mol}^{-1}$. Since there is only an influence on termination mechanism, according to Liu, the overall reaction rate constant can be calculated with kinetic data from other literature sources (Table 1 ). The resulting $k_{1}$ which considers the shear rate dependency, according to Liu, is significant smaller than the $k_{1}$ determined in this investigation with the Rheo NMR measurement (Table 2).

Conversely, the value of the $k_{1}$ calculated without a dependency of the fluid dynamics (data of Table 1), coincides nearly with the $k_{1}$ determined by the Rheo NMR measurement. This implied that there is no dependency of the fluid dynamics on the polymerization kinetics of MMA polymerization under the given conditions at a shear rate of $\dot{\gamma} \quad 128 \mathrm{~s}^{-1}$.

\section{Conclusion}

In this manuscript a temperature controlled Rheo NMR measuring cell was introduced. The heating is realized by a hot nitrogen stream, which flows through small axial channels in the wall of the outer cylinder. To control the temperature a thermo couple was implemented and calibrated. As a model system the free radical polymerization of MMA in solution was carried out under shear. An evaluation routine was proposed to determine the overall reaction rate constant $k_{1}$. The result was compared to literature data with and without shearing. This demonstrated that $k_{1}$ measured with the Rheo NMR cell matches with $k_{1}$ from literature without shearing. A dependency of the fluid dynamics on the polymerization kinetics could not be observed in the investigated parameter space for this model system. However, other authors describe the influence of fluid dynamics, for example for the polymerization of styrene. In future, other systems shall be inves tigated with the Rheo NMR measuring cell and checked whether there is an influence on polymerization kinetics.

\section{Acknowledgements}

The authors acknowledge the instrumental facility Pro $^{2}$ NMR financed by DFG, Dr. Dieter Gross and the Bruker BioSpin GmbH and the co workers of the NMR group in CIW of KIT.

\section{References}

[1] A. Kumar, S. Gupta, R. Mohan, Effect of shear rate on the rate of radical polymerization of methyl methacrylate, Eur. Polym. J. 16 (1) (1980) 710.

[2] M. Vicevic, K. Novakovic, K. Boodhoo, A. Morris, Kinetics of methyl methacrylate free radical polymerisation in the spinning disc reactor, in: 17th International Congress of Chemical \& Process Engineering (CHISA), Prague, Czech Republic, 2006.

[3] M. Vicevic, K. Novakovic, K. Boodhoo, A. Morris, Kinetics of styrene free radical polymerisation in the spinning disc reactor, Chem. Eng. J. 135 (1) (2008) 7882.

[4] K.V.K. Boodhoo, R.J. Jachuck, Process intensification: spinning disc reactor for condensation polymerisation, Green Chem. 2 (2000) 235244.

[5] K. Boodhoo, R. Jachuck, Process intensification: spinning disk reactor for styrene polymerisation, Appl. Therm. Eng. 20 (12) (2000) 11271146.

[6] Z. Liu, R. Kádár, M. Kind, Hydrodynamic activation of the batchpolymerization of methyl methacrylate in a taylor-couette reactor, in: Macromolecular Symposia, Vol. 302, Wiley Online Library, 2011, pp. 169178. 
[7] P.T. Callaghan, Rheo-nmr: nuclear magnetic resonance and the rheology of complex fluids, Rep. Prog. Phys. 62 (4) (1999) 599.

[8] J.D. Seymour, B. Manz, P.T. Callaghan, Pulsed gradient spin echo nuclear magnetic resonance measurements of hydrodynamic instabilities with coherent structure: taylor vortices, Phys. Fluids 11 (5) (1999) 11041113.

[9] G. Odian, Principles of Polymerization, John Wiley \& Sons, 2004.

[10] S. Chandrasekhar, The stability of viscous flow between rotating cylinders, in: Proceedings of the Royal Society of London a: Mathematical, Physical and Engineering Sciences, vol. 246, The Royal Society, 1958, pp. 301311.

[11] J. Cole, Taylor-vortex instability and annulus-length effects, J. Fluid Mech. 75 (01) (1976) 115.

[12] C.D. Andereck, S. Liu, H.L. Swinney, Flow regimes in a circular couette system with independently rotating cylinders, J. Fluid Mech. 164 (1986) 155183.

[13] U. Eichhoff, K. Zick, P. Callaghan, Nmr velocity mapping and rheo-nmr in complex liquids, Ukrayins' kij Fyizichnij Zhurnal (Kyiv) 56 (8) (2011) 793797.
[14] A. Esser, S. Grossmann, Analytic expression for taylor couette stability boundary, Phys. Fluids 8 (7) (1996) 18141819.

[15] Z. Liu, Einfluss der Vermischung auf chemische Reaktionen im Taylor-Couette Reaktor am Beispiel der Polymerisation von Methacrylat, KIT Scientific Publishing, 2013.

[16] F.M. Lewis, M.S. Matheson, Decomposition of aliphatic azo compounds, J. Am. Chem. Soc. 71 (2) (1949) 747748.

[17] S. Beuermann, M. Buback, T.P. Davis, R.G. Gilbert, R.A. Hutchinson, O.F. Olaj, G.T. Russell, J. Schweer, A.M. van Herk, Critically evaluated rate coefficients for free-radical polymerization, 2. propagation rate coefficients for methyl methacrylate, Macromol. Chem. Phys. 198 (5) (1997) 15451560.

[18] P. Penchev, Capacity flow method for determination of propagation and termination rate constants in radical polymerization, Die Makromol. Chem. 177 (2) (1976) 413428.

[19] A. Racina, Ph.D. thesis, Vermischung in Taylor-couette Stromung, vol. 2008, Karlsruhe Univ. Diss., 2008. 


\section{Repository KITopen}

Dies ist ein Postprint/begutachtetes Manuskript.

Empfohlene Zitierung:

Laryea, E.; Schuhardt, N.; Guthausen, G.; Oerther, T.; Kind, M.

Construction of a temperature controlled Rheo-NMR measuring cell - Influence of fluid dynamics on PMMA-polymerization kinetics.

2018. Microporous and mesoporous materials, 269

doi:10.5445/IR/1000069524

Zitierung der Originalveröffentlichung:

Laryea, E.; Schuhardt, N.; Guthausen, G.; Oerther, T.; Kind, M.

Construction of a temperature controlled Rheo-NMR measuring cell - Influence of fluid dynamics on PMMA-polymerization kinetics.

2018. Microporous and mesoporous materials, 269, 65-70.

doi:10.1016/j.micromeso.2017.03.038 